\title{
Research and Application of Public Traffic Intelligent Dispatching Based on Dynamic Programming Algorithm
}

\author{
Sixian Niu \\ School of Information Engineering, Baise University, Baise 533000, China;
}

\begin{abstract}
Keywords: Dynamic programming algorithm, traffic congestion, OD pair, multi-time dynamic traffic
\end{abstract} assignment.

\begin{abstract}
Because the static traffic assignment can not perfectly reflect status of traffic congestion in actual case and the model of dynamic traffic assignment may cause a huge computation cost, the article combines features of static traffic assignment and the dynamic one and has Dial' algorithm based multi-time dynamic assignment to get a balance between precision of description and computation cost. According to the method in the article, corresponding heuristic procedure is proposed, and it is also examined by numerical example of traffic assignment in situation such as network with different linear and nonlinear traveling function, network with link capacity constraint, one origin one destination and one origin several destinations OD pair paradigm. And it also proposes fitting function proximately reflecting overloaded condition in BPR paradigm and compares assignment results of BPR function with methods from other studies to prove practicality and correctness of multi-time assignment based on Dial's theory in the article..
\end{abstract}

\section{Introduction}

The urban city has played a very important role in human's living, social activities, business operation, material feeding and leisure entertainment, while traffic is treated as foundation of whole city operation. And as one of necessary premises in the development of society, urban influences every field in our life [1].

The United Nations Population Fund (UNPF) delivers a report in 2007 that the world is experiencing an unprecedented urbanization and developing countries and areas are even more so. And in 30 years of the early 21th century, it is estimated that the whole population of city in Asia will grow by more than double [2]; in Africa area, it is about one point five times. In 2008, allegedly, the whole population around the world has exceeded total population in rural area, that is to say there will be more than half living in the city.

China is quickly experiencing urbanization, where 18 million annual rural populations get into the city. Although urbanization rate currently accounts for only $40 \%$, at current peace, the urbanization rate in future will exceed more than $55 \%$ and it will account for $75 \%$ by 2050 [3]. There will be tens of millions of rural population getting into the city. China has more than 660 cities, in the near future more than half of population will be the urban resident and there will be 83 cities which have more than 7.5 million populations.

One can not live without commutation in our daily life. Generally, with the increase of population, traffic demand will go up as well, particularly in the urban areas. And with continuous development of society and improvement of people's livelihood [4], owning a private car has become a universal phenomenon. The burgeoning developments of automobile retail industry are also accompanied by the phenomenon, which gives rise to an increasing number of private cars. Meanwhile, measures such as road expansion and traffic capacity increasing can not keep up with the pace of traffic demand. Then conflict between traffic demand and supply inevitably appeared, which leads to social issues such as traffic accident, traffic delay and environmental issues. Traffic congestion, which is treated as origin of problems, is a big trouble not only in developing countries but also in those developed ones. And with deterioration of urban traffic congestion and traffic disorder, it has had a huge negative effect on the modern city construction and development of economy. 


\section{The basic methods of static traffic assignment}

Traffic Congestion. Traffic congestion caused serious effects in economy, society and environment fields, including fuel waste produced by delay and low velocity and environment contamination generated by frequent firing-accelerating-braking. What's more, because of congestion, aggressive or angry behavior by a driver of an automobile or other motor vehicle may occurred, and it is easy to induce accidents and serious impacts on quality of people's life and even lead to economic depression, to some extent. The Texas Transportation Institute estimates that, in 2000, the 75 largest metropolitan areas experienced 3.6 billion vehicle-hours of delay, resulting in 5.7 billion U. S. gallons (21.6 billion liters) in wasted fuel and $\$ 67.5$ billion in lost productivity [5], or about $0.7 \%$ of the nation's GDP. It is also estimated that the annual cost of congestion for each driver was approximately $\$ 1,000$ in very large cities and \$200 in small cities [6]. Traffic congestion is increasing in major cities and delays are becoming more frequent in smaller cities and rural areas.

Algorithms for static traffic assignment. The method can also be called as $0-1$ method, which is widely used to have static traffic assignment. In the process of the method, road weights are usually valued as coefficient, that is, it postulates traffic load of link has nothing to do with average velocity of vehicles. And for once the process assigns all of OD pair traffic flux into the shortest path, which means other paths have no assignment.

Determining step length of iteration, which ought to make, satisfies the equation:

$\sum_{a}\left(y_{a}^{k}-x_{a}^{k}\right) c_{a}^{k}\left[x_{a}^{k}+\lambda\left(y_{a}^{k}-x_{a}^{k}\right)\right]=0,0 \leq \lambda \leq 1$

Finding new starting node of iteration by the equation:

$x_{a}^{k+1}=x_{a}^{k}+\lambda\left(y_{a}^{k}-x_{a}^{k}\right)$

Denoting arbitrary decimal number by $£$, and it satisfies the restraint, iteration is terminated, else go to Step2. The restraint is as follow:

$$
\frac{\sqrt{\sum_{a}\left(x_{a}^{k+1}-x_{a}^{k}\right)^{2}}}{\sum_{a} x_{a}^{k}}<\varepsilon
$$

F-W method can be easily compiled by computer programming, so it plays an important role in solving traffic assignment issues. While the converging process in the method will be stagnant with the iteration continues. What's more, the method is significantly affected by initial solution, degree of congestion and termination rule of iteration process.

\section{Method Based on Dial's Algorithm}

Traffic impedance is one of the most important parameters in traffic assignment and transportation planning. It reflects all kinds of factors that are concerned by travelers in traffic mode selection. Traffic impedance is usually defined as time travelers spend in their trips and it is analyzed in the model under various conditions.

In modeling time-varying flows on traffic networks, the travel time of the link is often treated as a function of the number of vehicles on the link. That is, when traffic enters into link at time $t$, let the time taken to traverse it be the equation as follow:

$\tau(t)=f(x(t))$

If vehicles enter only at one side of link and they exit only at the other side. That is, no vehicle starts or terminates its journeys anywhere along the link, then conservation is shown as follow:

$x(t)=x(0)+\int_{0}^{t} u(w) d w-\int_{0}^{t} v(w) d w$

Basing on the travel time models which are widely used in the traffic assignment model, the variation relationship between outflow and travel time can be given in a condition where the inflow with time changes. Relational expressions between inflow and outflow and generalized expressions can also be given in its corresponding model of travel time of link.

Numerical examples: There are 4 numerical examples comprise this part. In the first example, the impedance function $\mathrm{z}(\mathrm{t})=\mathrm{a}+\mathrm{m}(\mathrm{t})$ will be used to update impedance of link and method proposed in 
former part will be used to solve traffic assignment without capacity constraint. In the second one, the impedance function $\mathrm{z}(\mathrm{t})=\mathrm{a}+\mathrm{t} 2(\mathrm{t})$ will used as well. Link capacity constraint will also be taken into account and some assumptions about capacity of link will be given so that reality of traffic condition may to some extent simulated approximately. In the third example, one to more OD pair paradigm will be solved and illustrated. And the last one used by Kong et al. will be solved by the method based on Dial's algorithm so that a comparison between Kong's method and the method proposed in the article can be shown by the analysis.

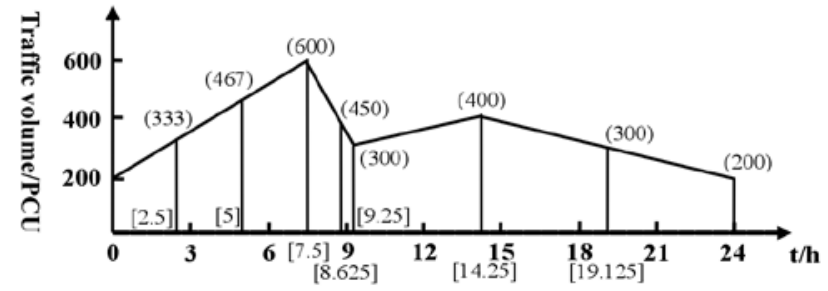

Fig. 1 Variety regulation of traffic flow in network I

Fig. 1 shows the regulation of variation of traffic volume in Network I. And we can divide time into 8 periods according to Fig.1, because there are 4 stable variations of traffic volume can be observed and some of them can be further on subdivided into several stages according to its variation rate of traffic volume.

Thus we can calculate traffic volume in each period, which can be shown in Tab. l.

Table 1 The traffic flow of different time

\begin{tabular}{cc}
\hline Period $i$ & Traffic volume $q(i)$ \\
\hline 1 & 666 \\
2 & 1000 \\
3 & 1334 \\
4 & 591 \\
5 & 421 \\
6 & 1575 \\
7 & 1706 \\
8 & 1216 \\
\hline
\end{tabular}

In Fig.2, it assumes that the distance among nodes in network I has been given. And origin node and destination are marked 1 and 16 respectively. In order to facilitate the illustration of heuristic procedure, several notes are needed to be shown, which are exhibited as follow:

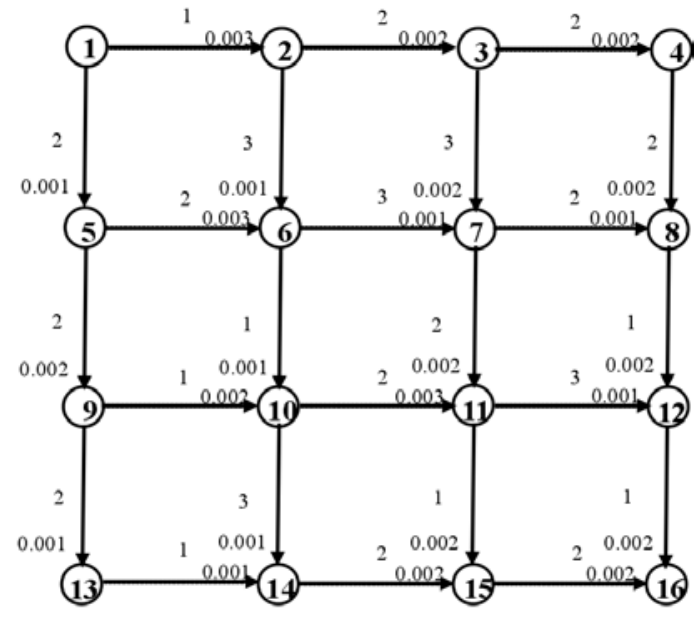

Fig. 2 Network I.

The example one and example two depict the traffic condition that travelers have trips from the same origin node to the same destination node and their choice may have interaction with each other. That is akin to saying that there are two ways starting from place A to another one B:a road with long distance and a short one having a bridge on it. It is common sense that travelers will generally treat the second one as their choice due to its economical time-use. But if there are something wrong with the bridge on the second road and it is articulated that the number of vehicles passing through the bridge at the same time is limited, the time passing through the bridge hence may increased, which means the 
travel time of the second road will increase. With the increasing number of travelers getting into the second road, the queuing up time in area near the bridge is increased as well, which can be to some extent regarded as congestion. And finally due to the congestion the travel time of second road will be larger than necessity time of the first road traveling from A to B. The first road becomes more attractive for travelers who want to get to $\mathrm{B}$, which explains the words "the path having the shortest distance does not necessarily mean it has the shortest travel time".

In the third example, one to more OD pair paradigm is solved and illustrated in the article. The example simulates the network which has several travelers starting from the same start node and getting to different destinations. Travelers' path choice in the network may have interactions with each other during the trip, which like one to one OD pair paradigm can make the shortest path or link on the network become time-waste one and make some remote roads more attractive. The difference of explanation between the former examples and the third one is that congestion not only makes original long distance path become more attractive, but also forced commuters driving to destination in their fixed path temporarily change the plan. And this can be used to explain some phenomena such as severe congestion in the holiday which makes latent destination (such as urban tourist spot) instantaneously attractive. Hence during the holiday, an increasing number of private vehicles getting to the new destination may result in congestion and bring big troubles for residents lived near the destination.

\section{Summary}

And according to result, it is also easy to get following conclusions: one of the most outstanding superiority of method based on Dial's algorithm proposed in the article, comparing with Kong's method, is that it approaches to the actual case. Reasons of such differences between two methods partly stems from shortcomings of AON assignment which is used in Kong's method.

\section{Acknowledgements}

The Key Project of Science and Technology of Henan Province: R\&D of the Low Carbon Transportation System (Grant No.12210231 0369). The Key Project of Science and Technology of Jiyuan City Henan Province: Development of the Transportation Data Exchange Sharing Platform in JiYuan City (Grant No.15013538).

\section{References}

[1] Lu H.P. Theory and Method in Transportation Planning [M]. (Bei Jing: Tsinghua University press, 2011). (In Chinese)

[2] Garcia R, Marin A. Network Equilibrium with Combined Modes: Models and Solution Algorithms. Transportation Research Pt.B, (2015) No. 39, p. 223-254.

[3] Ho H.W, Wong S.C, Loo B.P.Y Combined Distribution and Assignment Model for A Continuum Traffic Equilibrium Problem with Multiple User Classes. Transportation Research Pt.B, (2012) No. 40, p. 633-650.

[4] Sun H.J, Si B.F, Wu J.J. Combined Model for Flow Assignment and Mode Split in Two-modes Traffic Network. Journal of Transportation Systems Engineering and Information Technology, Vol. 8 (2015) No. 4, p. 77-82.

[5] J. Liu, E.L. Chen and Z.T. He: Journal of Shi Jia Zhuang Railway Institute (Natural Science), Vol. 22 (2014) No. 4, p.40-42. (In Chinese)

[6] Codina E, Garcia R, Marin A. New Algorithmic Alternatives for the OD Adjustment Problem on Traffic Networks. European Journal of Operational Research, (2016) No. 175, p. 1484-1500. 